\title{
An Improved Hybrid Color Image Watermarking under Various Attacks
}

\author{
C. N. Sujatha ${ }^{1}$, P. Satyanarayana ${ }^{2}$ \\ Associate Professor, ECE Department, SNIST, Hyderabad, India ${ }^{1}$ \\ Professor, ECE Department, AITS, Tirupati, India ${ }^{2}$
}

\begin{abstract}
This paper propose an improved hybrid color image watermarking algorithm using SVD, DCT and DWT. In this algorithm, SV's of transformed coefficients of RGB planes of host image using DCT and DWT are modified with that of color logo image at chosen scaling factors. Simulation results illustrate the good imperceptibility and robustness. The present algorithm bears desirable robustness on various attacks including Gaussian noise, salt \& pepper noise, compression, median filtering, average filtering, sharpening, rotation, histogram equalization, left and right cropping, top and bottom cropping and motion blurring attacks. Thus this algorithm is used for application of watermarking in image authentication and copyright protection. The performance analysis of this watermarking algorithm is measured by using MSE, PSNR and CF at various scaling factors.
\end{abstract}

Keywords: Watermark, DWT, DCT, SVD, PSNR, CF, Attacks.

\section{INTRODUCTION}

Digital image watermarking is the technique that embeds the image called watermark into the image or video objects. The embedded watermark can be detected to make an affirmation about the objects. Watermarking can be categorized as visible and invisible. Visible watermarking is important for protecting online resources from unauthorized reproduction, modification and transformation. Invisible watermarks can be detected by an authorized person, such watermarks are used for owner authentication and detecting unauthorized copying. Another criteria for the classification is based on the type of information needed by the detector, watermarking techniques are classified as Non-Blind watermarking which require the original image data for watermark extraction, Blind watermarking schemes require only secrete data.

Digital watermark embedding and extraction can be done in two ways: spatial domain and transform domain. In the spatial domain technique [1], the watermark can be inserted directly by manipulating the pixel intensity values of the original image/video frame. While in transform domain watermarking technique, digital image is transformed into frequency domain such that transform coefficients can be modified to hide the watermark [2-3]. Most commonly used transform domain techniques are Discrete Fourier transform, Discrete Cosine transform, Discrete Wavelet transform and Singular value decomposition.

Due to the availability of large storage devices like DVDs and USBs, storing and transferring of an image became very simple. This shows importance of developing a technique for copyright protection, authentication and protection against duplication. Simple distortions like noise concatenation, rotation, compression, filtering and

cropping of an image can prevent the detection of watermark. And in all frequency domain watermarking schemes, there is a discrepancy between robustness and transparency [4]. If the watermark is embedded in perceptually most significant components, the scheme would be robust to attacks but the watermark may be difficult to hide. On the other hand, if the watermark is embedded in perceptually insignificant components, it is easy to hide the watermark but the extraction of watermark from attacked watermarked image is difficult i.e the scheme may be least resistant to attacks. So it is desired to develop a novel robust watermarking algorithm using hybrid strategies which can resist a wide variety of attacks [5-6].

In this paper, we propose an imperceptible, robust and invisible non-blind color image watermarking scheme that incorporates multi resolution wavelet transformation, DCT and most powerful numerical analysis tool SVD.

\section{PROPOSED WATERMARKING SCHEME}

A novel hybrid color image watermarking have been proposed using three powerful transforms like DWT, DCT and SVD. In the proposed method, DWT is used to decompose the image into four subbands: LL, LH, HL and HH. In DWT based watermarking, the DWT coefficients are modified to embed the watermark data. As LL band holds the most of information of the image and the other bands contain the details of the image, any modification in LL band affects the quality of watermarked image. Because of the conflict between robustness and transparency, the modification at a given level is made in HL, LH, and HH subbands. Further more security is obtained by using other separable transform DCT and numerical transform SVD. 
DCT represents the image in low, mid and high frequency bands. We can embed the image data in the selected DCT transformed coefficients. SVD is a very convenient tool for watermarking in the hybrid domain using DWT and DCT [7-9]. The advantage of SVD based watermarking is that there is no need to embed all the singular values of a visual watermark. Depending on the magnitudes of the largest singular values, it would be sufficient to embed only a small set. In watermarking applications, SVD has following properties:

- SVD is able to efficiently represent the intrinsic algebraic properties of an image, where singular values correspond to the luminance of the image and singular vectors reflect geometry characteristics of the image.

- Singular values have good stability, which means small perturbation added to image will not significantly change the corresponding singular values.

- An image matrix has many small singular values compared with the first value. If these values are ignored it will have much effect on the quality of reconstructed image.

SVD provides good imperceptibility and robustness as it uses non fixed orthogonal bases and it preserves both oneway and non-symmetric properties which are not possible with other transformations. By adopting the merits of all three techniques, we presented a DWT-DCT-SVD based robust watermarking technique. Watermark embedding and extraction procedures are explored in the following sections.

\section{A. Watermark Embedding}

Let 'I' be the original color image and ' $\mathrm{W}$ ' be the color watermark. The embedding algorithm is shown in Fig. 1.

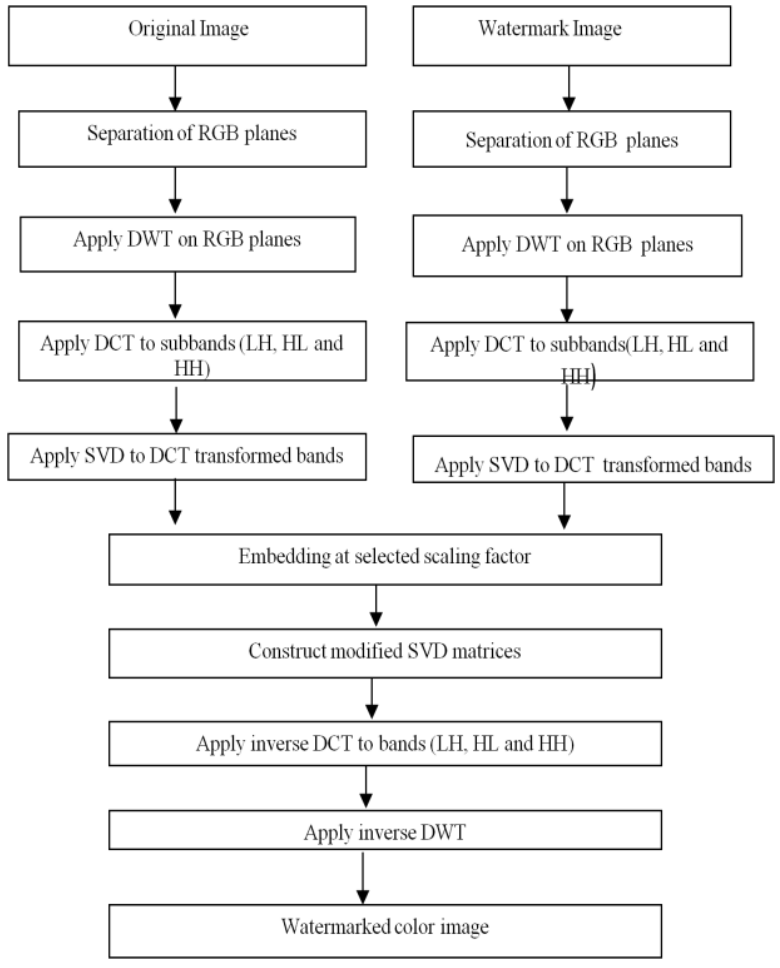

Fig. 1 Watermark Embedding algorithm
Original image 'I' and watermark 'W' both are decomposed into three color planes namely $\mathrm{R}, \mathrm{G}$ and $\mathrm{B}$. DWT is applied to each plane of image to get four subands. DCT is performed on horizontal, vertical and diagonal DWT coefficients and singular values are calculated using SVD technique. The watermark also processed through the same procedure. Singular values of the original image are added with the singular values of watermark image to form the singular values of watermarked image. Inverse DCT is performed followed by inverse DWT to get watermarked image ' $\mathrm{I}$ '.

\section{B. Watermark Extraction}

The extraction algorithm is shown in Fig. 2. First the watermarked image is divided into RGB planes. DWT is applied to each of these planes to select subbands. Then DCT and SVD are performed to obtain modified singular matrices. Singular values of watermark are extracted by subtracting the singular values obtained in the embedding process. IDCT and IDWT are applied to get embedded color watermark image ' $\mathrm{W}^{1}$ '.

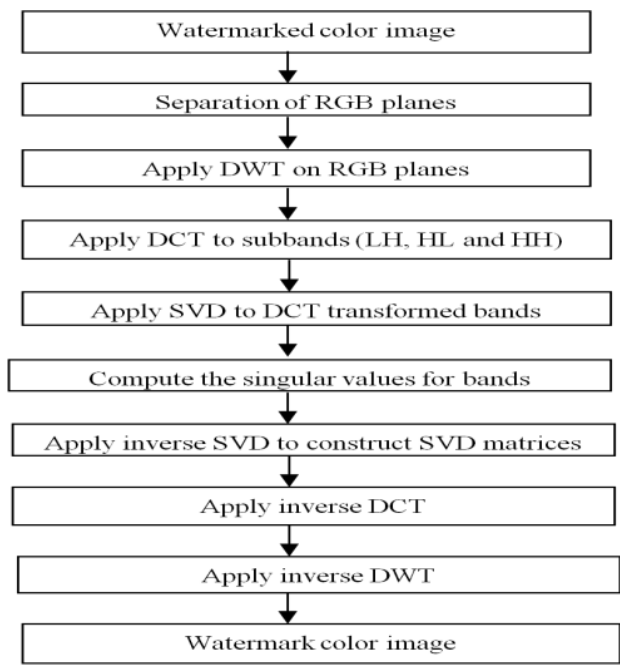

Fig. 2. Watermark extraction algorithm

\section{III.PERFORMANCE EVALUATION OF PROPOSED WATERMARKING SCHEME}

The simulation of proposed hybrid algorithm is done using MATLAB 7.14.0. The algorithm is tested on different formats of various standard test images Lena, peppers, sunset, balloon and autumn. In our experiments, original image 'I' and watermark image 'W' are of size $256 \times 256$ are used. Simulation results demonstrate the transparency of the proposed algorithm is estimated by measuring MSE and PSNR between original 'I' and watermarked images ' $\mathrm{I}^{1}$ ' and experimental results are listed in Table I.

The imperceptibility and robustness has been judged under various kinds of attacks. Fig. 3 shows the embedding of svu logo in autumn cover image at various scaling factors. Simulation results show the high quality watermarked image with high PSNR is obtained by embedding the logo at low range of scaling factors. But this affects the robustness of watermark. 


\section{A. Simulation Results}

TABLE II

PERFORMANCE METRICS FOR VARIOUS TEST IMAGES $(\alpha=0.01)$

\begin{tabular}{|l|l|l|}
\hline Original image & MSE & PSNR \\
\hline Peppers & 0.0139 & 66.7121 \\
\hline Lena & 0.0184 & 65.4929 \\
\hline Balloon & 0.0203 & 65.0614 \\
\hline Sunset & 0.0181 & 65.5658 \\
\hline Autumn & 0.0126 & 67.1101 \\
\hline
\end{tabular}

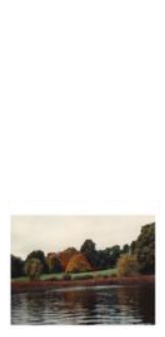

Cover Image
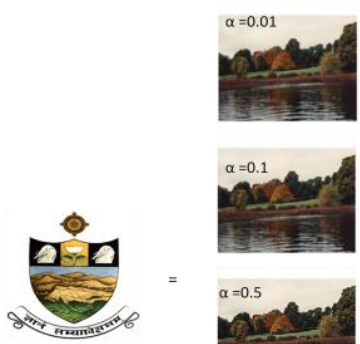

Watermark to be embedded
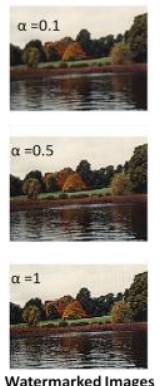

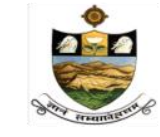

Extracted Watermark
Fig. 3 autumn image embedded with SVU logo at various scaling factors

\section{B. Effect of Attacks On Watermarked Color Image}

Attacks are factors that can degrade the strength of the watermark. Robustness is tested under 10 types of attacks: Gaussian noise, Salt \& Pepper noise, compression, median filtering, average filtering, rotation, sharpening, histogram equalization, motion blurring and cropping (left, right, top and bottom). Correlation factor is calculated to evaluate the robustness of the algorithm. The effect of attacks on PSNR and CF are shown in Table II. The retrieved watermarks with best quality from degraded watermarked image under various attacks are shown in Fig. 4 to Fig. 17.
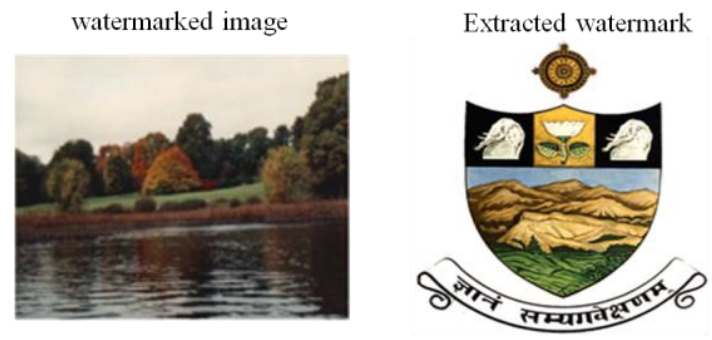

Fig. 4 watermarked and extracted watermark images without attacks
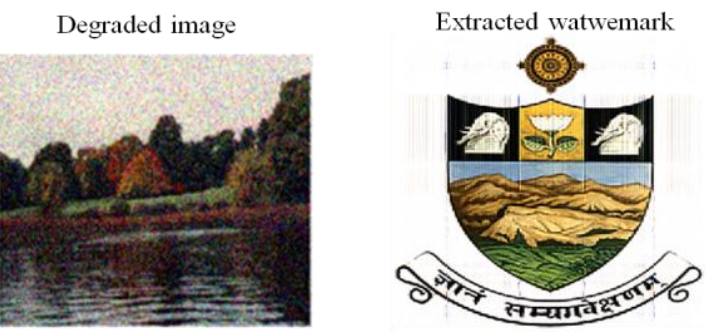

Fig. 5 Effect of Gaussian noise on watermarked image and extracted

$$
\text { watermark }
$$$$
\text { Copyright to IJARCCE }
$$

Copyright to IJARCCE
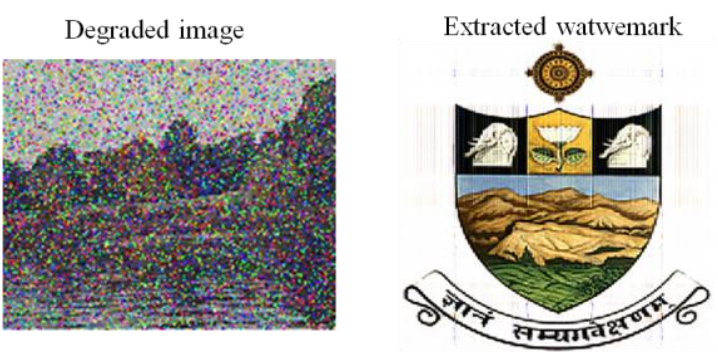

Fig. 6 Effect of Salt \& Pepper noise on watermarked image and extracted watermark
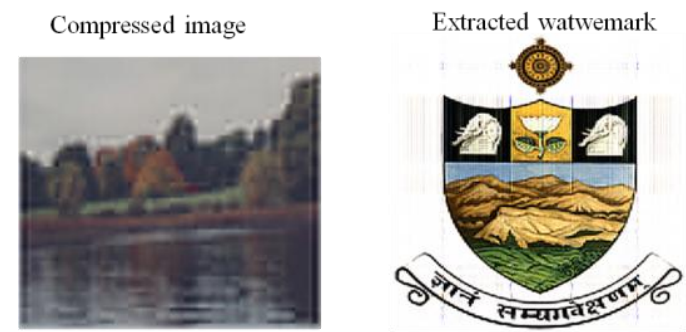

Fig. 7 Effect of Salt \& Pepper noise on watermarked image and extracted watermark
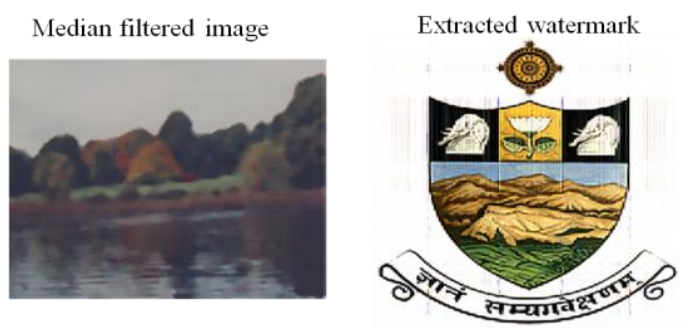

Fig. 8 Effect of Median filtering on watermarked image and extracted watermark
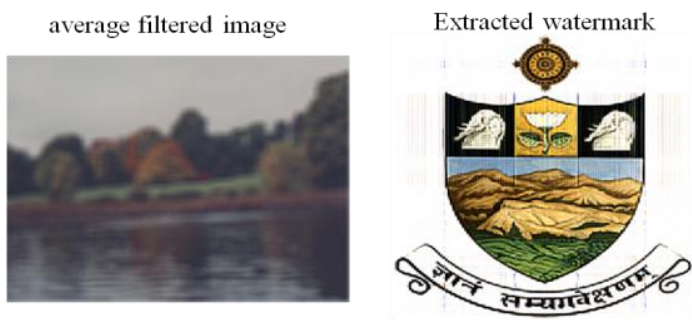

Fig. 9 Effect of Average filtering on watermarked image and extracted watermark
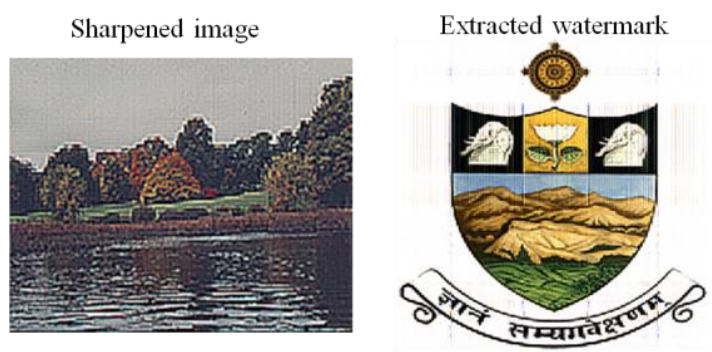

Fig. 10 Effect of Sharpening on watermarked image and extracted watermark 
rotated image
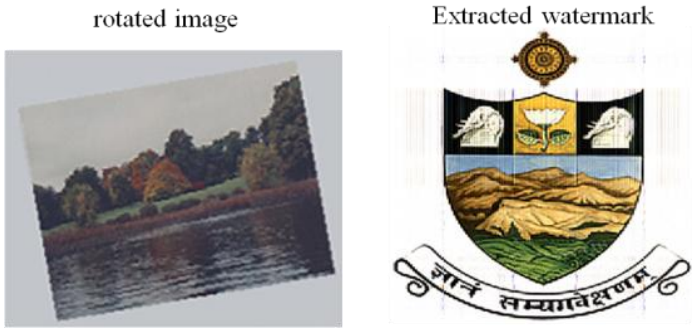

Fig. 11 Effect of Rotation on watermarked image and extracted watermark
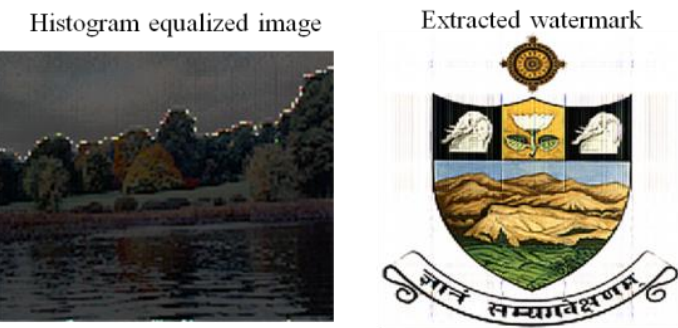

Fig. 12 Effect of Histogram equalization on watermarked image and extracted watermark
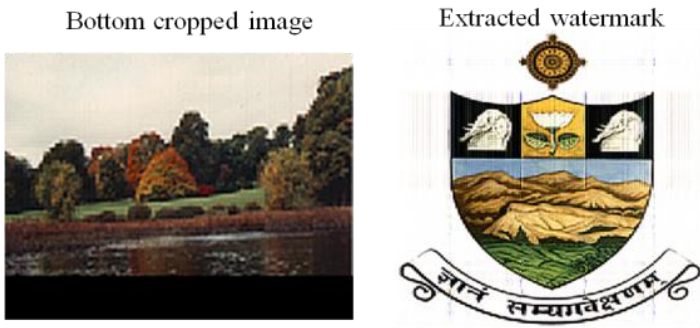

Fig. 13 Effect of Bottom cropping on watermarked image and extracted watermark
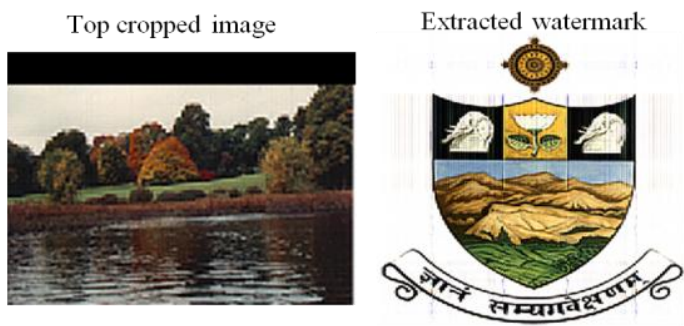

Fig. 14 Effect of Top cropping on watermarked image and extracted watermark
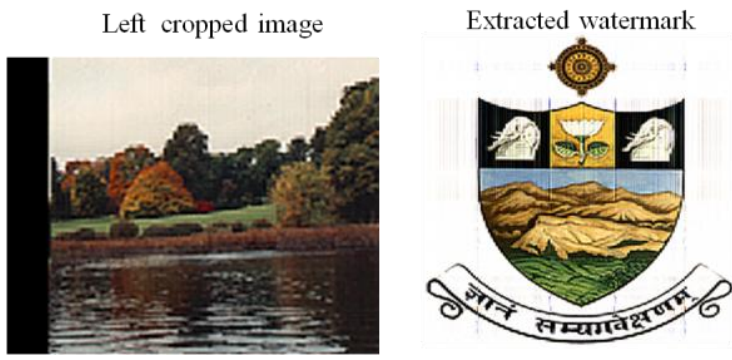

Fig. 15 Effect of Left cropping on watermarked image and extracted watermark
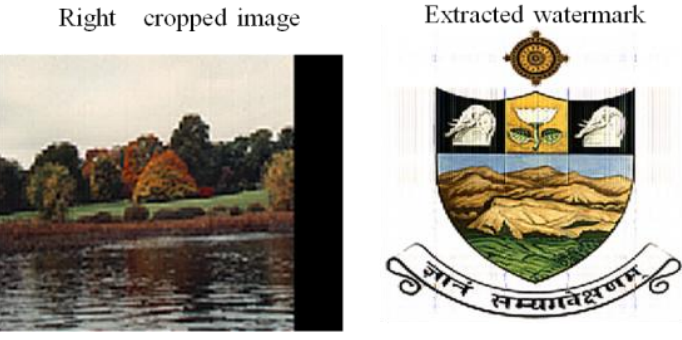

Fig. 16 Effect of Right cropping on watermarked image and extracted watermark
Motion blurred image

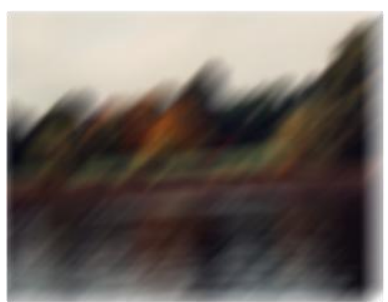

Extracted watermark

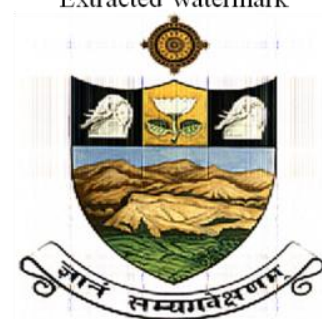

सम्यगत्व
Fig. 17 Effect of motion blurring on watermarked image and extracted watermark

TABLE II

PERFORMANCE METRICS UNDER VARIOUS ATTACKS

\begin{tabular}{|l|l|l|l|l|}
\hline \multirow{2}{*}{ attacks } & \multicolumn{2}{|c|}{ Without attack } & \multicolumn{2}{c|}{ With attack } \\
\cline { 2 - 5 } & PSNR & CF & PSNR & CF \\
\hline $\begin{array}{l}\text { Gaussian } \\
\text { noise }\end{array}$ & 62.4812 & 0.9797 & 21.6222 & 0.9797133 \\
\hline $\begin{array}{l}\text { Salt \& } \\
\begin{array}{l}\text { Pepper } \\
\text { noise }\end{array}\end{array}$ & 62.4812 & 0.9797 & 10.7006 & 0.9799189 \\
\hline compression & 62.4812 & 0.9797 & 19.3483 & 0.9795032 \\
\hline $\begin{array}{l}\text { Median } \\
\text { filtering }\end{array}$ & 62.4812 & 0.9797 & 21.1087 & 0.9795671 \\
\hline $\begin{array}{l}\text { Average } \\
\text { filtering }\end{array}$ & 62.4812 & 0.9797 & 25.0994 & 0.9795031 \\
\hline Sharpening & 62.4812 & 0.9797 & 9.0066 & 0.9800366 \\
\hline Rotation & 62.4812 & 0.9797 & 2.9243 & 0.9795475 \\
\hline $\begin{array}{l}\text { Histogram } \\
\text { equalization }\end{array}$ & 62.4812 & 0.9797 & 10.0670 & 0.9798538 \\
\hline $\begin{array}{l}\text { Right } \\
\text { cropping }\end{array}$ & 62.4812 & 0.9797 & 9.0585 & 0.9766723 \\
\hline $\begin{array}{l}\text { Left } \\
\text { cropping }\end{array}$ & 62.4812 & 0.9797 & 8.8622 & 0.9763842 \\
\hline $\begin{array}{l}\text { Top } \\
\text { cropping }\end{array}$ & 62.4812 & 0.9797 & 8.6378 & 0.9732001 \\
\hline $\begin{array}{l}\text { Bottom } \\
\text { cropping }\end{array}$ & 62.4812 & 0.9797 & 8.6085 & 0.9730237 \\
\hline $\begin{array}{l}\text { Motion } \\
\text { blurr }\end{array}$ & 62.4812 & 0.9797 & 22.0592 & 0.9794848 \\
\hline
\end{tabular}

The poor values of PSNR specify the amount of degradation on watermarked image. This improved algorithm can retrieve the true color watermark image from the highly distorted watermarked image with very little degradation. From the results, it has been observed that embedding of watermark at scaling factor above 0.1 will sustain to attacks. From the figures (Fig. 4 - 17), we find that our algorithm not only can successfully resist different kinds of attacks but can also restore watermark with high perceptual quality. We observed that correlation 
factor between original watermark and extracted watermark after applying attacks is larger if the scaling factor is high. The proposed hybrid algorithm demonstrates that when we increase the scaling factor, we get better performance especially in terms of robustness while keeping the image quality requirement at reasonable levels.

\section{IV.CONCLUSION}

In this paper, we emphasized on invisible color image watermarking that provides a comprehensive evaluation algorithm that embeds and extracts the color watermark image effectively. The embedded image is extracted with the help of original image; hence it is a non-blind color image watermarking. The simulation results show the high quality watermarked image with largest PSNR values obtained by embedding watermark image at lower values of scaling factor, but it affects the robustness of watermark. From the results of hybrid technique, we observed that watermarked image is robust against different kinds of attacks, by embedding watermark image at higher range of scaling factors and we can conclude that original and watermarked images are visually same. With this improved watermarking technique, large values of correlation factors are gained that demonstrate the similarity between embedded and extracted color logo images. The advantage of the present hybrid algorithm is that it can hide the color logo of equal size of original color image. It has very high data hiding capacity. Results prove that the proposed technique is robust against all sorts of attacks.

\section{REFERENCES}

[1] I. Nasir, Ying Weng and Jianmin Jiang, "A New Robust Watermarking Scheme for Color Image in Spatial Domain", $3^{\text {rd }}$ Int. IEEE conf. on SITIS, Shanghai, pp. 942-947, Dec. 2007.

[2] Ankita Durge and Alka Jaiswal, "Increase Security of Images from Attacks using Watermarking Technique", Int. J. of Scientific \& Engineering Research (IJSER), Vol. 3, Issue. 9, Sept. 2012.

[3] P.R. Reddy, M. V. N. K. Prasad and D. S. Rao, "Robust Digital Watermarking of Color Images under Noise Attacks", Int. J. of Recent Trends in Engineering, Vol. 1, No. 1, May 2009.

[4] Cheng-qun Yin, Li Li, An-qiang Lv and Li Qu, "Color Image Watermarking Algorithm Based on DWT-SVD", Proc. Of IEEE Int. Conf. on Automation and Logistics, Jinan, China, Aug. 2007.

[5] U.M. Gokhale and Y.V. Joshi, "A New Watermarking Algorithm Based on Image Scrambling and SVD in Wavelet Domain", ACEEE Int. J. on Network Security, Vol. 02, No. 03, July 2011.

[6] S. Sirmour and T. Archana, "A Hybrid DWT-SVD Based Digital Image Watermarking Algorithm for Copyright Protection", Int. J. of P2P Network Trends and Technology (IJPTT), Vol. 6, pp. 7-10, Mar. 2014.

[7] V. Santhi and T. Arunkumar, "DC coefficients Based Watermarking Tecnique for Color Images using Singular Value Decomposition", Int. J. of Computer and Electrical Engineering, Vol. 3, No.1, Feb. 2011.

[8] P. S. Murthy, R. Hima Bindu and P. R. Kumar, "A Novel SemiBlind Reference Color Image Watermarking using DWT-DCTSVD”, Int. J. of Computer Applications (IJCA), Vol. 53, No. 15, Sept. 2012.

[9] K. Chaitanya, E. S. Reddy and K. G. Rao, "Digital color Image Watermarking in RGB Planes using Singular DWT-DCT-SVD Coefficients", Int. J. of Computer Science and Information Technologies, Vol. 5, No.2, pp.2413-2417, Feb. 2011.

\section{BIOGRAPHIES}

Mrs. C. N. Sujatha, accomplished B.Tech (Electronics \& Communication Engineering, 2001), M.Tech (Electronic Instrumentation and Communication Systems, 2005) and at present Pursuing Ph.D. from S.V. University, Tirupati, A.P, India. Presently associated with SNIST, Hyderabad, Telangana as Associate Professor, Dept. of ECE. And presented papers in International and National conferences in various fields and published two papers in international journals. Research areas of Interests are Image \& Video Processing, Speech Processing \& Digital Signal Processing.

Dr. P. Satyanarayana, accomplished B.Tech (1976), M.Tech (1978), and Ph.D. in 1987 from S.V. University Tirupati. He also obtained his Post Doctoral Fellow in 1-D Signal Processing from Concordia University, Montreal, Canada. Currently associated with AITS, Tirupati, A.P., as Professor, Dept. of ECE. He worked as Head, Dept. of E.C.E, S.V. University, Tirupati, A.P., India. He visited many countries like Malaysia, U.S.A and other countries as visiting faculty. He is the member of expert committee of NBA and chairman, BOS (E.C.E) for P.G. in S.V. University, Tirupati and V.S. University, Nellore, A.P., India. He has presented 16 papers in International Journals like IEEE \& IETE and more than 30 papers in National Conferences. 